\title{
Energy-Optimal Velocity Profiles for Car-Like Robots
}

\author{
Pratap Tokekar, Nikhil Karnad and Volkan Isler
}

\begin{abstract}
For battery-powered mobile robots to operate for long periods of time, it is critical to optimize their motion so as to minimize energy consumption. The driving motors are a major source of power consumption. In this paper, we study the problem of finding velocity profiles for car-like robots so as to minimize the energy consumed while traveling along a given path.

We start with an established model for energy consumption of DC motors. We present closed form solutions for the unconstrained case and for the case where there is a bound on maximum velocity. We also study a general problem where the robot's path is composed of segments (e.g. circular arcs and line segments). We are given a velocity bound for each segment. For this problem, we present a dynamic programming solution which uses the solution for the single-constraint case as a subroutine. In addition, we present a calibration method to find model parameters. Finally, we present results from experiments conducted on a custom-built robot.
\end{abstract}

\section{INTRODUCTION}

In this work, we study the problem of energy efficient navigation. Specifically, we focus on car-like robots powered by DC motors. It is well-known that the energy consumption of a DC motor depends on the angular velocity and acceleration. We study the problem of computing the velocity profile of a robot so that it consumes a minimum amount of energy to travel along a given path.

Even though energy efficient navigation is a fundamental problem, it has received very little attention, and a comprehensive treatment is missing. Existing literature includes the work of Sun and Reif [1] who consider the problem of computing the optimal path over a terrain. Under the assumption that the friction coefficients are known across the terrain, they show how to compute a path that requires minimum energy to overcome frictional forces. This work generates the path but does not yield an optimal velocity and acceleration profile. In this sense, it is complementary to the present work.

In order to compute the velocity and acceleration profiles, power consumption needs to be modeled. Mei et al. [2] model the power consumption as a sixth-degree polynomial of the robot's speed using experimentally collected data. However, their model does not incorporate acceleration. More importantly, they use this model to compare velocity profiles but do not address the problem of computing an optimal profile.

Kim and Kim [3] find the optimal velocity profile for a robot moving on a straight line, when the total time to

P. Tokekar, N. Karnad and V. Isler are with the Department of Computer Science and Engineering, University of Minnesota, 200 Union Street SE, Minneapolis MN 55414 USA. e-mail: \{tokekar, karnad, isler\}@cs.umn.edu.

This work is supported by NSF projects 0916209,0917676 and 0936710. travel is fixed. However, this solution does not incorporate any bound on maximum velocity of the robot. In [4], they propose a rotational trajectory planner that minimizes the energy consumption. They do not present a systematic method to combine the solutions for translational and rotational trajectories. Thus, it is not clear if this approach yields an optimal solution. Wang et al. [5] studied the problem of finding a minimum energy trapezoidal velocity profile. As we will show shortly, trapezoidal profile itself is not optimal in terms of total energy consumption. In addition, they too do not consider any upper bound on the velocity of the robot. Further, their technique is only applicable for turn-in-placemove-forward type of motion for differential drives, and is not experimentally verified.

The rest of the paper is organized as follows: After presenting the formal problem statement and the energy model in Section II, we present a calibration procedure for estimating the parameters of the energy model in Section III. The optimal velocity profiles with and without a maximum velocity bound are derived in Sections IV and V respectively. The application of these results to compute the velocity profile over an entire path composed of multiple segments is presented in Section VI. Experiments on our custom-robot are presented in Section VII. We conclude with a discussion on the utility of our results in Section VIII.

\section{PROBlem Formulation}

We are given a path $\Pi$ along which the robot moves. Let $D$ be its total length. Since the motion of the robot is restricted to $\Pi$, we can represent the instantaneous position by a single parameter, $x(t)$. The forward velocity and acceleration of the robot are represented by $v(t)$ and $a(t)$ respectively. We define the state of the robot by $\mathbf{X}(t)=[x(t), v(t)]^{T}$. The state transition equation can be written as $\dot{\mathbf{X}}(t)=[v(t), a(t)]^{T}$.

The problem we address in this paper is to compute the velocity profile, $v(t)$ of the robot along $\Pi$ which minimizes the energy consumption for motion. We start with the energy consumption model in the next section.

\section{A. Energy Model}

We use the model described in [6] for energy consumption in a brushed DC motor. This detailed model considers the energy dissipated in the resistive winding, the energy required to overcome internal and load friction and the mechanical power delivered to the output shaft. The instantaneous current $i(t)$ in the motors is given by,

$$
i(t)=\frac{1}{K_{T}}\left[T_{F}+T_{L}+D_{f} \omega(t)+\left(J_{M}+J_{L}\right) \frac{d \omega(t)}{d t}\right]
$$


and the voltage $e(t)$ across the motor is given by,

$$
e(t)=i(t) R+K_{E} \omega(t)
$$

where $\omega(t)$ is the angular velocity of the motor, $K_{E}$ and $K_{T}$ are back-EMF and torque constants, $T_{F}$ and $T_{L}$ are internal and load frictional torques, $D_{f}$ is the internal damping force and $J_{M}$ and $J_{L}$ are motor and load moments of inertia.

Since linear velocity of the robot and angular velocity of the motor for a car-like robot are proportional to each other, we can rewrite Equations 1 and 2 to yield the energy consumption can be written as,

$$
\begin{aligned}
E= & \int_{0}^{t_{f}}[e(t) i(t)] d t \\
= & \int_{0}^{t_{f}}\left[c_{1} a^{2}(t)+c_{2} v^{2}(t)+c_{3} v(t)\right. \\
& \left.+c_{4}+c_{5} a(t)+c_{6} v(t) a(t)\right] d t
\end{aligned}
$$

where constants $c_{1}, \ldots, c_{6}$ are combinations of the motor parameters, and $v(t)$ and $a(t)$ are the linear velocity and acceleration of the robot obtained from $\omega(t)$ and the radius of the wheel. When the initial and final velocity values are the same for $\Pi$, the net contribution by the terms corresponding to $c_{5}$ and $c_{6}$ is zero. Hence, we can rewrite the energy model as,

$$
E=\int_{0}^{t_{f}}\left[c_{1} a^{2}(t)+c_{2} v^{2}(t)+c_{3} v(t)+c_{4}\right] d t .
$$

The constants $c_{1}, \ldots, c_{4}$ depend on the motor parameters which in turn depend on the robot design and the surface on which the robot is moving. These parameters can be obtained using the calibration procedure presented in Section III.

\section{B. Problem Statement}

Recall that $\Pi$ is a path of length $D$. The energy consumption for a velocity profile $v(t)$ traversing $\Pi$ is given by Equation 3. The final time $t_{f}$ can be fixed or kept free. We study three problems of increasing generality. For each of the problems, the objective is to minimize $E$ subject to the constraints given below:

Problem 1: There is no bound on the maximum velocity of the robot. The robot starts from and returns to rest i.e., the initial and final boundary conditions are given as $x(0)=0$, $v(0)=0$, and $x\left(t_{f}\right)=D, v\left(t_{f}\right)=0$.

Problem 2: The maximum velocity of the robot over $\Pi$ is bounded by $v_{m}$, i.e., $v(t) \leq v_{m}$ for $0 \leq t \leq t_{f}$. The initial and final velocities of the robot can be non-zero, i.e., $v(0)=$ $v_{0}$ and $v\left(t_{f}\right)=v_{f}$ subject to $v_{0}, v_{f} \leq v_{m}$.

Problem 3: $\Pi$ consists of $N$ segments made up of straight lines and curves. There is a separate velocity bound for each segment. For each segment $i$, we are given the velocity bound $v_{m}(i)$ and the distance to travel $D(i), 1 \leq i \leq N$. The objective is to find the optimal velocity profile over the entire path $\Pi$. The robot starts from rest and returns to rest at the end of the path.
The solutions for Problems 1, 2 and 3 are presented in Sections IV, V and VI respectively. We begin by describing the calibration procedure to determine the model parameters.

\section{CAlibration}

In this section, we describe a simple procedure to find the energy model (Equation 3) of the robot for a given flat surface. We use a custom-built robot (see Figure 1) for experiments. The robot is driven using two DC motors whose output shafts are connected together. It has car-like steering controlled by an independent servo motor. Separate batteries are used to drive the DC motors and power the rest of the electronics on the robot.

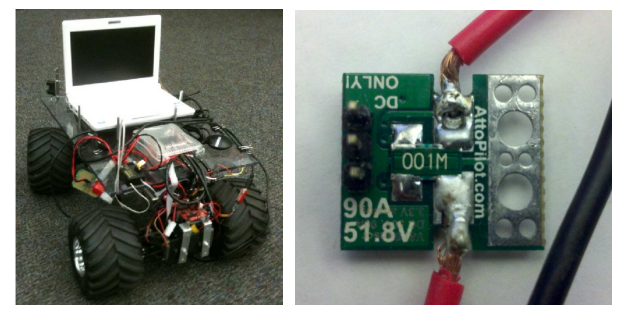

Fig. 1. Left: Custom-built robot used in our experiments. Right: Attopilot voltage and current measurement circuit from SparkFun Electronics.

\section{A. Experimental Setup}

Our method utilizes a simple current and voltage measurement circuit (Figure 1) connected between the output of the motor driver circuit and the motor. This circuit measures the current flowing through and the voltage across the motor. The linear velocity of the robot is measured using an optical encoder installed on one of its wheels. In the calibration procedure described next, the steering of the robot is set so that the robot moves in a straight line.

\section{B. Calibration Procedure}

We can write Equations 1 and 2 as,

$$
i(t)=b_{1}+b_{2} v(t)+b_{3} a(t), e(t)=b_{4}+b_{5} v(t)+b_{6} a(t)
$$

where $b_{1}, \ldots, b_{6}$ are linear combinations of the internal parameters of the motors. The calibration procedure to obtain the energy parameters consists of the following steps:

STEP 1: Move the robot at a constant velocity $\left(v_{\text {set }}\right)$ for some time interval. Log the current and voltage across the motor. Repeat for different $v_{\text {set }}$ values ranging from the minimum to the maximum achievable velocity for the robot. Figure 2(a) shows some of the actual profiles obtained during calibration for $v_{\text {set }}$ from $0.5 \mathrm{~m} / \mathrm{s}$ to $2.5 \mathrm{~m} / \mathrm{s}$.

STEP 2: Compute the average current and voltage for each of the above trials disregarding the initial acceleration phase. Using Equation 4, we can find the parameters $b_{1}, b_{2}$, $b_{4}$ and $b_{5}$ using least-squares linear fitting to the data (see Figure 2(b and c)).

STEP 3: To find the remaining two terms $b_{3}$ and $b_{6}$ in the model, we program the robot to move from rest at various set acceleration values $a_{\text {set }}$ for some interval of time (see Figure 2(d)). 

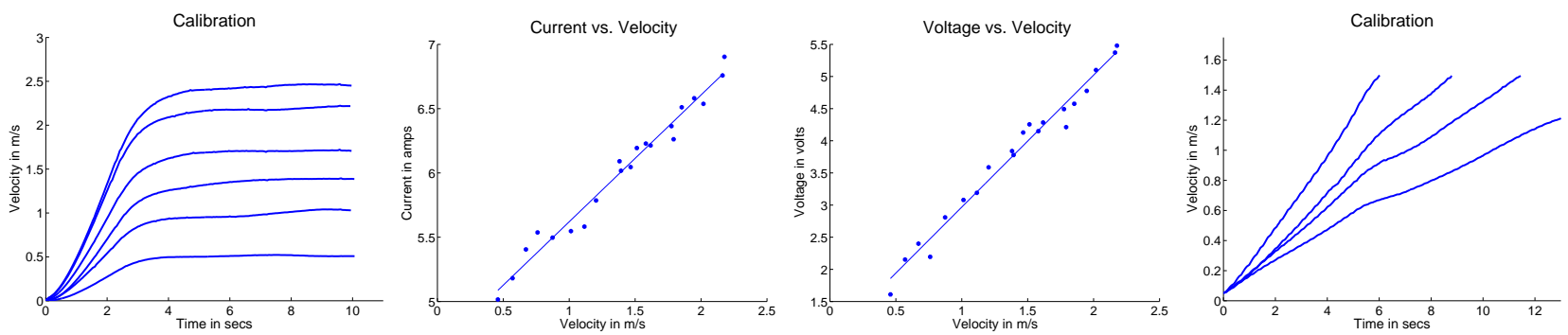

Fig. 2. Figures obtained during calibration on the corridor surface. Left to right: (a) The robot initially accelerates from rest to various set velocity values. The average current and voltage is computed for the region where the robot has reached $v_{\text {set }}$ (STEP 1). (b) Current consumption as a linear function of the velocity, when the motor is not accelerating (STEP 2). (c) Voltage applied to the motor as a linear function of the velocity, when the motor is not accelerating (STEP 2). (d) Calibration procedure to determine the parameter $c_{1}$ in the energy model. The robot is accelerated with various set acceleration values $a_{\text {set }}$ while logging current and voltage values (STEPS 3 and 4).

STEP 4: Compute the values of $b_{3}$ and $b_{6}$ by substituting $a_{\text {set }}$ and $b_{1}, b_{2}, b_{4}$ and $b_{5}$ values obtained above in Equation 4 and taking the average of all the readings.

STEP 5: Finally, the required parameters $c_{1}, \ldots, c_{4}$ in the model used throughout the paper (Equation 3 ) can then be calculated as $c_{1}=b_{3} b_{6}, c_{2}=b_{2} b_{5}, c_{3}=b_{1} b_{5}+b_{2} b_{4}$, and $c_{4}=b_{1} b_{4}$.

Using the above procedure, we calibrated our robot on a building corridor surface. The parameters obtained after calibration were: $c_{1}=17.75, c_{2}=1.16, c_{3}=10.46$ and $c_{4}=4.70$ SI units. The rest of the paper uses these parameters for illustrations.

\section{OPTIMAL UNCONSTRAINED VELOCITY PROFILE}

In this section, we present the solution to Problem 1. We first state the necessary conditions and present the closed form solution for the optimal velocity profile. Then, we discuss and provide insights for the structure of the optimal profile. Finally, we compare the optimal profile with the commonly-used trapezoidal velocity profile. Due to space constraints, we omit some proofs in this conference version, and direct the readers to the technical report [7].

\section{A. Solution to Problem 1}

When there is no bound on the maximum velocity, the Hamiltonian [8] for this problem can be obtained as,

$$
\begin{aligned}
H(\mathbf{X}(t), a(t), \boldsymbol{\lambda}(t), t) & =c_{1} a^{2}(t)+c_{2} v^{2}(t)+c_{3} v(t) \\
& +c_{4}+\lambda_{1}(t) v(t)+\lambda_{2}(t) a(t)
\end{aligned}
$$

where $\lambda_{1}(t)$ and $\lambda_{2}(t)$ are the Lagrange multipliers and acceleration $a(t)$ is the control.

The three necessary conditions for $a^{*}(t)$ to optimize the Hamiltonian for all time $t \in\left[0, t_{f}\right]$ are given as,

$$
\dot{\mathbf{X}}^{*}(t)=\frac{\partial H}{\partial \boldsymbol{\lambda}}, \quad \dot{\boldsymbol{\lambda}}^{*}(t)=-\frac{\partial H}{\partial \mathbf{X}}, \quad 0=\frac{\partial H}{\partial a}
$$

Applying these necessary conditions, we can solve the resulting partial differential equations for the optimal control and states to get,

$$
\begin{aligned}
& a^{*}(t)=k s_{1} e^{k t}-k s_{2} e^{-k t} \\
& v^{*}(t)=s_{1} e^{k t}+s_{2} e^{-k t}-\left(\frac{c_{3}+s_{3}}{2 c_{1}}\right) \\
& x^{*}(t)=\frac{s_{1} e^{k t}}{k}-\frac{s_{2} e^{-k t}}{k}-\left(\frac{c_{3}+s_{3}}{2 c_{1}}\right) t+s_{4} .
\end{aligned}
$$

where $k=\sqrt{\frac{c_{2}}{c_{1}}}$ and $s_{1}, \ldots, s_{4}$ are constants.

We can solve for $s_{1}, \ldots, s_{4}$ in terms of the final time $t_{f}$ by substituting the boundary conditions given in Problem 1 for $v^{*}(t)$ and $x^{*}(t)$. We obtain,

$$
\begin{aligned}
& s_{1}=-\frac{D k}{k t_{f}+e^{k t_{f}}\left(k t_{f}-2\right)+2}, \quad s_{2}=s_{1} e^{k t_{f}}, \\
& s_{3}=2 c_{1}\left(s_{1}+s_{2}\right)-c_{3}, \quad s_{4}=-\frac{s_{1}-s_{2}}{k} .
\end{aligned}
$$

By substituting in Equation 8 we obtain the optimal velocity profile $v^{*}(t)$ in terms of $t_{f}$ as,

$$
v^{*}(t)=D \sqrt{\frac{c_{2}}{c_{1}}}\left(\frac{\left(1+e^{k t_{f}}-\left(e^{k\left(t_{f}-t\right)}+e^{k t}\right)\right)}{k t_{f}+e^{k t_{f}}\left(k t_{f}-2\right)+2}\right) .
$$

Since the final time is free, it can be solved for using the additional boundary condition (known as the transversality condition) given by,

$$
H\left(\mathbf{X}^{*}\left(t_{f}\right), a^{*}\left(t_{f}\right), \boldsymbol{\lambda}^{*}\left(t_{f}\right), t_{f}\right)=0 .
$$

Substituting Equations 7-9 and 10 above results in,

$$
\left(D \frac{c_{2}}{c_{1}}+2\right)\left(1-e^{k t_{f}}\right)+\sqrt{\frac{c_{4}}{c_{1}}} k t_{f}\left(1+e^{k t_{f}}\right)=0,
$$

which is an equation in single variable $t_{f}$ (all other terms are constant) and can be solved using any solvers. (We used MATLAB's solve function). Alternatively, if the final time is fixed, we can directly substitute this given value in Equation 11 to find $v^{*}(t)$.

Figure 3 shows the optimal velocity and the corresponding optimal control profile obtained for traveling distances of 5, 35, 70 and $100 \mathrm{~m}$ using Equation 11. It is worth noting that the optimal profile reaches the same peak velocity and does not go faster even if the distance to travel increases. This peak velocity value represents the energy trade-off 

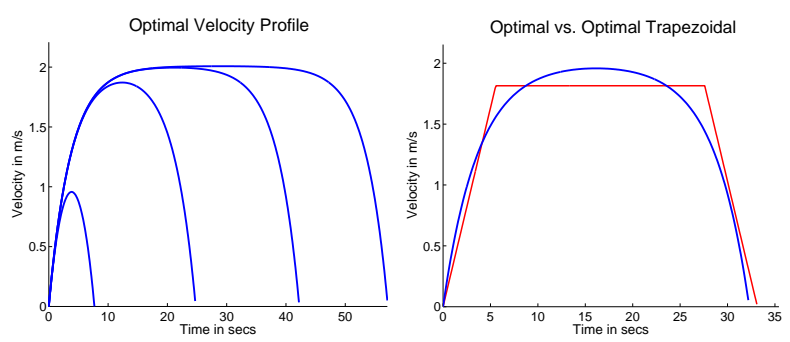

Fig. 3. Left: The optimal velocity profiles for distances $D=$ $5,35,70,100 \mathrm{~m}$ have a similar structure reaching the same peak velocity at $t=t_{f} / 2$. Right: Comparison between optimal trapezoidal profile and general optimal profile. The general optimal profiles gains larger savings during accelerations and decelerations.

between moving faster (and consequently for a lesser time) and moving slower (and for longer times).

Figure 3 shows the general optimal profile and optimal trapezoidal profile computed for traveling a distance of $D=50 \mathrm{~m}$. The general optimal profile we compute gains higher savings with respect to the trapezoidal profile while accelerating and decelerating. For example, the optimal profile yields $1.94 \%$ savings when traveling $1 \mathrm{~m}$, while the savings drop to $0.32 \%$ when $D=100 \mathrm{~m}$ for the parameters calculated on our custom robot. These figures are highly system-specific. In situations where the robot has to frequently stop, following an optimal profile would result in more energy savings and a longer lifetime. The velocity profile computed in this work is guaranteed to minimize the energy consumption for the stated assumptions.

\section{General solution InCORPORATING MAXIMUM VELOCITY BOUND}

The optimal profile given in Section IV does not satisfy any bound on the maximum velocity imposed by the physical limitations of the robot. In this section, we solve for the optimal velocity profile with a bound on the maximum achievable velocity $v(t) \leq v_{m}$. Additionally, the initial and the final velocities of the robot can be non-zero i.e., $v(0)=v_{0}$ and $v\left(t_{f}\right)=v_{f}$ subject to $v_{0}, v_{f} \leq v_{m}$.

In the energy model, we assumed that the initial and final velocity are the same. Hence, the resulting profile from Problem 2 when initial and final velocities are different would not be energy-optimal. However, such a case is only used as a subroutine for solving part of Problem 3. In Problem 3, the first and the last segments have zero initial and final velocities respectively, and hence the resulting solution obtained by using Problem 2 as a subroutine remains optimal. We now derive the analytical solution for Problem 2 by first discussing the possible structures of an optimal profile.

\section{A. Structure of optimal profile}

Depending on the value of $v_{m}$ and $D$, the optimal velocity profile can belong to one of the following two cases:

CASE 1: Unconstrained solution does not violate $v(t) \leq v_{m}$

In this case, the optimal velocity profile computed in Section IV is a valid solution for the constrained case. This can happen when $v_{m}$ is larger than the peak velocity reached by the unconstrained solution i.e., $v_{m} \geq \sqrt{\frac{c_{4}}{c_{2}}}$. The details are presented in [7]. Additionally, when the distance to travel $D$ is so small that the velocity profile does not change much (see Figure 3 when $D=5 \mathrm{~m}$ ), unconstrained solution would be feasible and hence, optimal.

The analytical solution presented for the unconstrained form was derived for zero initial and final velocities. By following a similar process, we can extend this to non-zero initial and final values to get,

$$
\begin{aligned}
& s_{1}=\frac{\left(v_{0}-v_{f}\right)\left(1-e^{-k t_{f}}-k t_{f}\right)+D k\left(1-e^{-k t_{f}}\right)}{e^{k t_{f}}\left(2-k t_{f}\right)+e^{-k t_{f}}\left(2+k t_{f}\right)-4}, \\
& s_{2}=\frac{\left(v_{0}-v_{f}\right)\left(1-e^{k t_{f}}+k t_{f}\right)-D k\left(1-e^{k t_{f}}\right)}{e^{k t_{f}}\left(2-k t_{f}\right)+e^{-k t_{f}}\left(2+k t_{f}\right)-4}, \\
& s_{3}=2 c_{1}\left(s_{1}+s_{2}\right)-c_{3}-v_{0}, \quad s_{4}=-\frac{s_{1}-s_{2}}{k} .
\end{aligned}
$$

CASE 2: Unconstrained solution violates $v(t) \leq v_{m}$

In this case, the solution consists of unconstrained $\mathcal{U}$ $\left(v(t)<v_{m}\right)$ and constrained $\operatorname{arcs} \mathcal{C}\left(v(t)=v_{m}\right)$ joined together at corner points. The optimal solution must be a $\mathcal{U}-\mathcal{C}-\mathcal{U}$ sequence having corner points at times $t=t_{1}$ and $t=t_{f}-t_{2}$, along with its degenerate cases $(\mathcal{U}-\mathcal{C}, \mathcal{C}-\mathcal{U}, \mathcal{C})$ when either or both of $t_{1}$ and $t_{2}$ equal to 0 . This can be shown easily by proving that all other sequences consume more energy than a $\mathcal{U}-\mathcal{C}-\mathcal{U}$ sequence. The full proof is presented in [7].

We now show how to obtain the solution for this case in closed form. Specifically, we show how to obtain $v^{*}(t)$ for the unconstrained and constrained arcs and compute the corner points $t_{1}$ and $t_{f}-t_{2}$.

\section{B. Solution}

We begin by writing the velocity constraint in the form of state inequality $\bar{S}=\left(v(t)-v_{m}\right) \leq 0$. The state inequality $\bar{S}$ is converted into a control equality $\bar{S}^{(1)}$ and interior point constraint $G$ by differentiating $\bar{S}$ once, leading to $\bar{S}^{(1)}=\dot{v}(t)=u$ and $G=\xi\left(v(t)-v_{m}\right)$. The Hamiltonian is augmented with the control equality constraint between $\left[t_{1}, t_{f}-t_{2}\right]$ and is given by $\hat{H}=H+\mu(t) a(t)$, where $\mu(t)$ is the slack variable associated with the control constraint and $H$ is given by Equation 5 .

The three necessary conditions given in Equation 6 are used to obtain the optimal profile in the time interval $\left[0, t_{1}\right]$ and $\left[t_{f}-t_{2}, t_{f}\right]$. On the constraint boundary, i.e. $t \in\left[t_{1}, t_{f}-\right.$ $t_{2}$, the following necessary conditions must hold,

$$
\dot{\mathbf{X}}^{*}(t)=\frac{\partial \hat{H}}{\partial \boldsymbol{\lambda}} \quad \dot{\boldsymbol{\lambda}}^{*}(t)=\frac{\partial \hat{H}}{\partial \mathbf{X}} \quad 0=\frac{\partial \hat{H}}{\partial a}
$$

Additionally, on the two corners $\left(t=t_{1}, t=t_{f}-t_{2}\right)$, the following conditions must hold for the optimal solution [9],

$$
\begin{gathered}
H\left(t_{1}^{+}\right)=H\left(t_{1}^{-}\right)+\left[\frac{\partial G}{\partial t}\right]_{t_{1}}, \boldsymbol{\lambda}\left(t_{1}^{+}\right)=\boldsymbol{\lambda}\left(t_{1}^{-}\right)-\left[\frac{\partial G}{\partial \mathbf{X}}\right]_{t_{1}}^{T} \\
H\left(\left(t_{f}-t_{2}\right)^{+}\right)=H\left(\left(t_{f}-t_{2}\right)^{-}\right) \\
\boldsymbol{\lambda}\left(\left(t_{f}-t_{2}\right)^{+}\right)=\boldsymbol{\lambda}\left(\left(t_{f}-t_{2}\right)^{-}\right)
\end{gathered}
$$


Using these conditions, we can solve for the optimal control and velocity profiles in terms of the constants for the off-boundary exponential curves and times $t_{1}, t_{2}$ and $t_{f}$. The optimal velocity profile is given by,

$v^{*}(t)=\left\{\begin{array}{r}s_{1}\left(e^{k t}+e^{k\left(2 t_{1}-t\right)}-\left(1+e^{2 k t_{1}}\right)\right)+v_{0}, \\ 0 \leq t \leq t_{1} \\ v_{m}, \\ t_{1} \leq t \leq t_{f}-t_{2} \\ s_{2}\left(e^{-k\left(t_{f}-t-2 t_{2}\right)}+e^{k\left(t_{f}-t\right)}-\left(1+e^{2 k t_{2}}\right)\right)+v_{f}, \\ t_{f}-t_{2} \leq t \leq t_{f} .\end{array}\right.$

We can obtain the values of these constants and times using the initial and final conditions, the transversality condition given in Equation 12, and the interior point constraint $v^{*}(t)=v_{m}$ in $t_{1} \leq t \leq t_{f}-t_{2}$ to give,

$$
\begin{gathered}
s_{1}=-\frac{\left(v_{m}-v_{0}\right)}{\left(e^{k t_{1}}-1\right)^{2}}, \quad s_{2}=-\frac{\left(v_{m}-v_{f}\right)}{\left(e^{k t_{2}}-1\right)^{2}}, \\
t_{1}=\frac{1}{k} \ln \left(\frac{c_{4}+c_{2} v_{m}^{2}-2 c_{2} v_{0} v_{m}}{c_{4}-c_{2} v_{m}^{2}}\right. \\
\left.+\frac{2\left(c_{2} v_{m}\left(c_{4}-c_{2} v_{0} v_{m}\right)\left(v_{m}-v_{0}\right)\right)^{\frac{1}{2}}}{c_{4}-c_{2} v_{m}^{2}}\right), \\
t_{2}=\frac{1}{k} \ln \left(\frac{c_{4}+c_{2} v_{m}^{2}-c_{2} v_{f} v_{m}}{c_{4}-c_{2} v_{m}^{2}}\right. \\
\left.+\frac{2\left(c_{2} v_{m}\left(c_{4}-c_{2} v_{f} v_{m}\right)\left(v_{m}-v_{f}\right)\right)^{\frac{1}{2}}}{c_{4}-c_{2} v_{m}^{2}}\right) .
\end{gathered}
$$

The final time can then be calculated by using the total distance to travel and the distances traveled in the two exponential curves.

$$
t_{f}=t_{1}+t_{2}+\frac{x^{*}\left(t_{f}-t_{2}\right)-x^{*}\left(t_{1}\right)}{v_{m}} .
$$

It is easy to see that if $v_{0}$ or $v_{f}$ is equal to $v_{m}$, then $t_{1}=0$ or $t_{2}=0$ respectively.
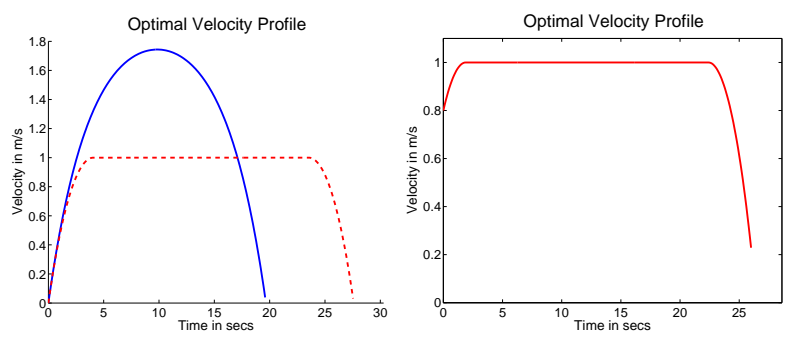

Fig. 4. Left: Optimal Velocity profile obtained for maximum velocity bound $v_{m}=1 \mathrm{~m} / \mathrm{s}$. Right: Optimal velocity profile with $v_{0}=0.8 \mathrm{~m} / \mathrm{s}$, $v_{m}=1 \mathrm{~m} / \mathrm{s}$ and $v_{f}=0.1 \mathrm{~m} / \mathrm{s}$ for traveling $25 \mathrm{~m}$.

Figure 4 (left) shows the optimal velocity profile obtained for traveling a distance of $25 \mathrm{~m}$ with the maximum velocity bound set to $v_{m}=1 \mathrm{~m} / \mathrm{s}$ and $v_{0}, v_{f}=0$, along with the corresponding solution when there is no constraint on the maximum velocity. Figure 4 (right) shows the optimal velocity profile obtained for the traveling a distance of $25 \mathrm{~m}$, with velocity bound $v_{m}=1 \mathrm{~m} / \mathrm{s}$ and initial and final velocities $v_{0}=0.8 \mathrm{~m} / \mathrm{s}$ and $v_{f}=0.2 \mathrm{~m} / \mathrm{s}$ respectively.

\section{Vi. Optimal Profile over Multiple Segments}

We now use the general solution given above to solve for the problem of finding an optimal velocity profile over a path consisting of $N$ segments (see Figure 5). Depending on the surface on which a car-like robot operates, the maximum feasible speed without either slipping or overturning for each segment will depend on the radius of the segment. Hence, for each segment, we fix a maximum allowable velocity $v_{m}(i)$ and are given a distance $D(i), 1 \leq i \leq N$ to travel.

The robot initially starts at and returns to rest, i.e., $v_{0}(1)=$ 0 and $v_{f}(N)=0$. The velocities $v_{0}(i)$ and $v_{f}(i)$ can be nonzero for all other intermediate segments. We can compute the optimal velocity profile for a given segment, if we know the $v_{0}(i)$ and $v_{f}(i)$ that the optimal velocity profile uses.
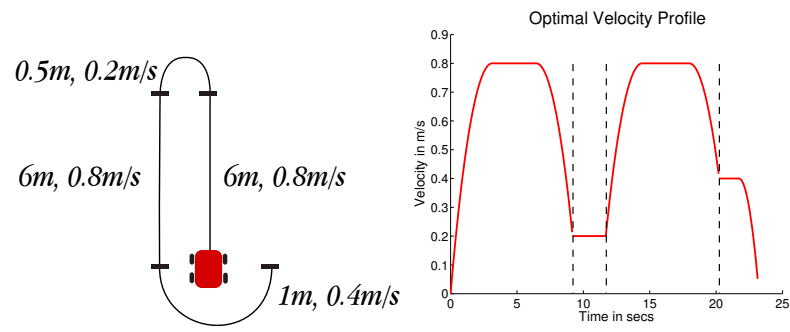

Fig. 5. Left: Typical path for a robot composed of two straight line segments and two turns of different radii. Segments have different maximum allowable velocities, depending on their radii. Right: Optimal velocity profile with different bounds for different segments. The given path consists of 4 segments with bounds $v_{m}=\{0.8,0.2,0.8,0.4\} \mathrm{m} / \mathrm{s}$ and distances $D=\{6,0.5,6,1\} m$

Let $V_{\max }$ be the maximum of $\left\{v_{m}(i), \forall i\right\}$ over all segments. We then discretize the velocity space at the segment boundary into $M$ equal partitions $v^{(k)}=\frac{k}{M} V_{\max }, 0 \leq k \leq$ $M$. Let $C\left(v^{(k)}, i\right)$ be the cost to reach the velocity $v^{(k)}$ at the $i^{\text {th }}$ segment boundary. Let $E\left(v_{0}, v_{m}, v_{f}\right)$ be a function which gives the energy consumption for an optimal velocity profile in a segment starting with $v_{0}$ and ending with $v_{f}$. If either $v_{0}>v_{m}$ or $v_{f}>v_{m}$ then the function returns the cost as $E\left(v_{0}, v_{m}, v_{f}\right)=\infty$. We can then use the following recurrence for the $i^{t h}$ segment boundary and $1 \leq k \leq M$ :

$C\left(v^{(k)}, i\right)=\min _{0 \leq j \leq M}\left(C\left(v^{(j)}, i-1\right)+E\left(v^{(j)}, v_{m}(i), v^{(k)}\right)\right)$

Since the robot initially starts from rest, we have:

$$
C\left(v^{(k)}, 0\right)= \begin{cases}0 & k=0, \\ \infty & 1 \leq k \leq M .\end{cases}
$$

The solution can be obtained by backtracking from $C\left(v^{(0)}, N\right)$ and finding optimal segment boundary velocity values. The optimal velocity profile can then be constructed using these optimal boundary velocity values to find individual segment profiles.

Figure 5 (right) shows the optimal velocity profile obtained using above dynamic programming for a path consisting of 4 segments. The velocity bounds for these segments are $v_{m}=\{0.8,0.2,0.8,0.4\} \mathrm{m} / \mathrm{s}$. This solution is optimal up to numerical precision along the segment boundaries only and exact everywhere else. 


\section{EXPERIMENTS}

To test the validity of our results, we performed experiments using our custom robot. The experiments were performed on the smooth corridor surface for which the robot was calibrated. We first computed the analytical solution for the velocity profile to travel the given distance. This profile was then sampled at $10 \mathrm{~Hz}$ and values were stored in a lookup table.

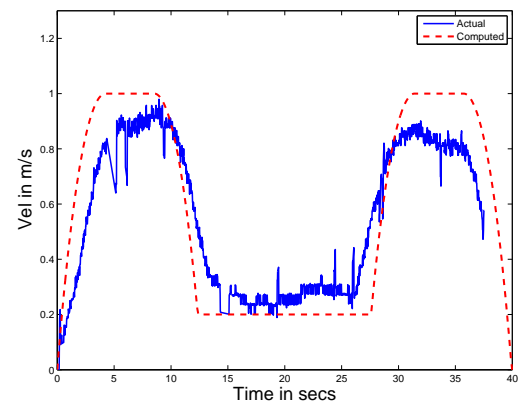

Fig. 6. Optimal velocity profile executed by the robot for multiple segments. The dashed curve shows the optimal profile computed using the dynamic programming solution for segments with $D=10,3,10 \mathrm{~m}$ and maximum velocity constraints $v_{m}=1,0.2,1 \mathrm{~m} / \mathrm{s}$.

Figure 6 shows the optimal profile computed using the dynamic programming solution presented in Section VI, for three segments with distances $D=10 \mathrm{~m}, 3 \mathrm{~m}$ and $10 \mathrm{~m}$ and maximum velocity constraints as $v_{m}=1 \mathrm{~m} / \mathrm{s}, 0.2 \mathrm{~m} / \mathrm{s}$ and $1 \mathrm{~m} / \mathrm{s}$. The computed velocity profile is shown as a dashed curve. The total energy consumed over the entire profile was $595 \mathrm{~J}$. As can be seen, the actual profile executed has small deviations arising due to noise and disturbances on the surface. In this work, we pre-compute the optimal trajectory for the robot. A useful extension to this could be to design an optimal velocity feedback controller which minimizes the energy consumption.

We compare the energy consumption of our optimal profile with two commonly-used trapezoidal profiles. The maximum speeds for these profiles are chosen as $1 \mathrm{~m} / \mathrm{s}$ and $2 \mathrm{~m} / \mathrm{s}$, so that the robot covers the same distance taking more and less time than the optimal respectively. We perform these comparisons for $D=20 \mathrm{~m}$ and $D=45 \mathrm{~m}$.
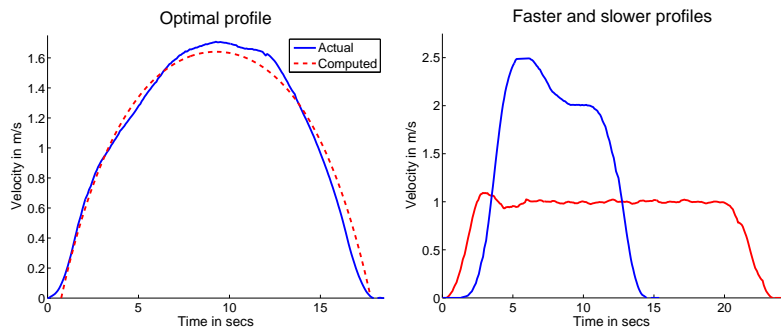

Fig. 7. Left: Optimal velocity profile executed by the robot for traveling $20 \mathrm{~m}$ in $18.4 \mathrm{~s}$ while consuming $296 \mathrm{~J}$ energy. The optimal profile is shown as dashed. Right: Sub-optimal velocity profiles executed by the robot for traveling $20 \mathrm{~m}$ at maximum set velocities of $1 \mathrm{~m} / \mathrm{s}$ and $2 \mathrm{~m} / \mathrm{s}$. The energy consumption for these profiles is $303 \mathrm{~J}$ and $319 \mathrm{~J}$.
Figure 7 shows the optimal, slower and faster velocity profiles executed by the robot in the corridor. The optimal profile computed is also shown in Figure 7 as dashed. Table I shows the comparison of the energy consumption for all the trials conducted. As we can observe, the optimal profile consumes lesser energy than the two sub-optimal profiles. Also, the energy savings become more significant as the distance traveled increases.

TABLE I

ENERGY CONSUMPTION DURING EXPERIMENTS

\begin{tabular}{|c|c|c|c|}
\hline $\mathrm{D}(m)$ & $E_{\text {opt }}(J)$ & $E_{\text {slow }}(J)$ & $E_{\text {fast }}(J)$ \\
\hline \hline 20 & 296 & 303 & 319 \\
\hline 45 & 656 & 694 & 696 \\
\hline
\end{tabular}

\section{CONCLUSION}

In this work, we studied the problem of computing a velocity profile for a car-like robot so as to minimize the energy consumed while traveling along a given path on a flat surface. We presented closed form solutions for two cases: no constraints on the robot's speed, and a single upperbound on the speed. We also studied a general case where the robot's path is composed of segments and we are given a speed upper-bound for each segment. For example, the robot's path can be composed of circular and straight-line segments. When the robot is following the circular sub-path, the maximum speed it can achieve can be less than the maximum speed for the straight component. These bounds may vary depending on the surface properties. For this general case, we presented a dynamic programming solution which builds on the solution for the single-bound case.

In addition, we presented a calibration procedure for obtaining robot's internal parameters related to energy consumption. We demonstrated the utility of the calibration procedure and the algorithms presented in the paper with experiments performed on a custom-built robot.

\section{REFERENCES}

[1] Z. Sun and J. Reif, "On finding energy-minimizing paths on terrains," IEEE Transactions on Robotics, vol. 21, no. 1, pp. 102-114, 2005.

[2] Y. Mei, Y. Lu, Y. Hu, and C. Lee, "Energy-efficient motion planning for mobile robots," in IEEE International Conference on Robotics and Automation, vol. 5, 2004.

[3] C. Kim and B. Kim, "Minimum-energy translational trajectory generation for differential-driven wheeled mobile robots," Journal of Intelligent and Robotic Systems, vol. 49, no. 4, pp. 367-383, 2007.

[4] - "Minimum-Energy Rotational Trajectory Planning for Differential-Driven Wheeled Mobile Robots," in Proc. 13th Int. Conf. on Advanced Robotics, 2007, pp. 265-270.

[5] G. Wang, M. Irwin, P. Berman, H. Fu, and T. La Porta, "Optimizing sensor movement planning for energy efficiency," in Proceedings of the 2005 international symposium on Low power electronics and design. ACM, 2005, p. 220.

[6] DC Motors, Speed Controls, Servo Systems: An Engineering Handbook. Electro-Craft Corporation, 1977.

[7] P. Tokekar, N. Karnad, and V. Isler, "Energy optimal velocity profiles for car-like robots," Department of Computer Science \& Engineering, University of Minnesota, Tech. Rep. 11-003, February 2011.

[8] D. Kirk, Optimal Control Theory: An Introduction. Prentice Hall, 1970.

[9] D. Hull, Optimal control theory for applications. Springer Verlag, 2003. 